\title{
Routing strategy using local information based on a two layer cellular automaton model
}

\author{
$\underline{\text { B. Zhu }}^{a}{ }^{a}$ and R. P. Liem ${ }^{\mathrm{a}} \odot$ \\ ${ }^{\mathrm{a}}$ Hong Kong University of Science and Technology, Hong Kong \\ Email: bzhuaf@connect.ust.hk
}

\begin{abstract}
A reliable and efficient traffic network model is required to study urban traffic congestion, which has increasingly become a global concern recently. The model can then be used to emulate different policy scenarios to assess different mitigation strategies, which will be very useful to policy- and decision makers. In this work, we develop a physical traffic model that can be used to investigate the intrinsic property of city traffic under different human decisions and driving behaviors. Cellular automaton is one of the most commonly used traffic network model. At its simplest stage, however, it can only model a one-dimensional problem. The Biham-Middleton-Levine (BML) extends the capability of the cellular automaton model to model a two-dimensional traffic network problem. However, it can only model two directions: rightward and downward. Directly modeling the four directions of traffic using the BML model causes jamming and gridlock problem. Thus, the existing traffic network models have not been sufficiently capable of modeling the traffic situation realistically. In this work, we propose a two-layer network modeling to address this intrinsic gridlock problem, where each layer is modeled based on the cellular automaton approach. The model is developed on a two-dimensional $L^{2}$-square lattice system, and users can specify the vehicle density prior to running the simulation. A moving strategy is then derived for each vehicle based on the origin and destination cell locations, where the shortest path is typically assumed. One of the key purposes of this work is to present the city traffic in a physical way to investigate the inertial characteristics of city traffic and to help bridge the gap between the simplified cellular automaton models and the complexity of real-world traffic. As such, we incorporate driving behavior modeling into the two-layer network system by introducing a flexibility index. Essentially, this index denotes the probability that a driver can deviate from the predefined shortest path when congestion occurs, i.e., when the next cell in its intended moving direction is occupied by another vehicle. The rationale behind this feature is that in real situation, drivers have the option to take an alternative path. We perform a number of traffic simulations to demonstrate the derived model and to gain insight into the effect of flexibility on the overall traffic flow. In particular, we vary the lattice size (by varying L) and the traffic density $\rho$, which will determine the number of vehicles to be simulated. Each vehicle is assigned a random origin-destination pair, and the corresponding moving strategy is then determined. By plotting the average vehicle speed as a function of vehicle density, we can find the phase transition point, where the traffic changes from a free-flow state to a congestion state. Our results show that when drivers are more flexible, the onset of congestion state is delayed to a higher density value. In other words, for the same density value, introducing a higher flexibility results in a higher average speed. This suggests that the vehicles can reach the destination faster, even if they need to cover a longer travel distance. This hypothesis is confirmed as we observe the effects of flexibility on the total distance traveled and the total number of completed journeys. Overall, our simulation results are consistent with the real traffic situations. This model can be further extended to mimic the traffic network more realistically by introducing more complexity in the system (e.g., the system lattice layout). As such, we will be able to evaluate some scenarios that the existing traffic models can not emulate accurately, such as the effect of traffic disruptions on the overall network flow.
\end{abstract}

Keywords: Traffic model, network modeling, cellular automaton, driving behavior 


\section{INTRODUCTION}

Urban traffic congestion is a serious issue which affects people's daily life on a global scale. It does not only affect people's travel and work, but also increases the risk of environmental pollution. To study traffic congestion and to assess the right mitigation strategies to implement, first and foremost we need to have a reliable traffic model. In this paper, we mainly focus on developing a physics-model to study the dynamics of traffic network and how human iteraction will influence the traffic. Such a model is used to measure some quantities that can characterize the traffic flow, such as the average speed, travel time, waiting time, and traffic flow [Maerivoet and De Moor (2005); Chen et al. (2012)]. Cellular automata (CA) is a class of computationally efficient microscopic traffic flow models, which is commonly used in traffic simulations and studies |Maerivoet and De Moor (2005)]. To study the urban transportation network, Angel et al. (2005) and Chopard (2012) used the Biham-Middleton-Levine (BML) model, which is an extension of the CA model to two-dimensional space. The BML model is a two-direction traffic network model which can describe the green wave and stop-andgo phenomena, and can be used to investigate the traffic congestion characteristics of the traffic network on a global scale. The BML model can also emulate the two phases of traffic flow, namely the free-flow and congestion states. However, the BML model faces some limitations, as it can only move towards the right, or downwards [Nagatani (1993); Angel et al. (2005)]. A direct extension of the BML model to include four directions will cause a gridlock, which is defined as a situation when the routing strategies of more than one vehicle will clash and prevent further movement of the vehicles locally. This situation can cascade into a global gridlock, which will terminate the traffic network modeling. Consequently, the BML model can not avoid intrinsic congestion, and thus can not reflect the actual real-world traffic situation. Many researches focus on specific scenarios when using CA to study the physical features of traffic network. Multi-lane models and simulations are typically modeled based on the CA model /Wagner et al. (1997); Davis (2004)]. Davis (2004) simulated the model with dual-lane highway and a single-lane highway with an on-ramp to make the model more realistic. Based on the multi-lane model, Tang et al. (2007), Li et al. (2006), and Zheng (2014) used the CA model to study the lane changing behavior of vehicles. In 2006, Li et al. (2006) used the fundamental CA model to study the lane-changing behavior of vehicles, and addressed the problem on the behavior of agressive lane changing on a two lane traffic model. In 2018, Tang et al. (2018) also generated a CA model in one juction with traffic lights to study the electrical vehicle's lane chaging behavior. Because of its microsopic view, CA can be used to describe the behaviour of individual vehicles. Burstedde et al. (2001) and Weifeng et al. (2003) employed the CA model to study the pedestrian movement and behaviors. Here we take a more global approach and generate our model based on the CA programming paradigm from statistical physics. In particular, we seek to explore the features of driver path selection behaviors in a scalable traffic network. The complexity of real traffic limits how much a physical model can represent the real traffic conditions. In this work, we propose a two-layer network approach to address the aforementioned limitation of the BML model and make the model more realistic. In particular, we adopt a two-layer approach to model the four directions of vehicle movement, to better mimic how vehicles actually operate in real traffic situation. A mechanism is introduced to avoid traffic gridlock. This two-layer network model helps bridge the gap between simplified CA models and the complexity of real-world traffic. Once established, this model will open door to model the actual road network, traffic disruptions such as road blocking and accidents.

The derived model will then be used to study the effect of driving behavior on the traffic congestion. Driving behavior is one of the most important factor that will influence the traffic efficiency [Zamith et al. (2015)]. The path selection, which reflect a driving behavior, is known to have a major impact on transit time [Golledge (1995)]. The transit time reflects the traffic efficiency; when we maximize the traffic efficiency, the traffic congestion is consequently reduced. Many works have been done on path routing strategies. Oliveira and Carneiro (2014) presented the hot-spot placement strategy based on people's daily trajectory. Yeung and Saad (2012) worked on increasing the network efficiency by importing non-shortest path.

To model the driving behavior, we introduce a flexibility index, which represents the driver's tendency to change the routing strategy when the original route is blocked, albeit temporarily. In the modeling, we first assume that all drivers will travel by choosing the shortest path (in terms of distance) between the origin and destination. With the flexibility included in the model, the driver is given the option to pick a direction other than the pre-computed shortest path. In other words, they can deviate from the shortest path when encountering traffic congestion. The rationale behind this concept of flexibility is that in real traffic situation, some people have the tendency to choose the direction with fewer vehicles to avoid congestion.

In this paper, we present our proposed a two-dimensional routing strategy that can mimic the four-directional vehicle movements. This is achieved by introducing a two-layer network model based on the CA concept, 
and this model is categorized as a microscopic traffic flow model. In addition to modeling the four directional movements of the vehicles, this two-layer approach can also help avoid gridlock in the modeling. To further reflect the real traffic situation, the derived model can include a flexibility measure to reflect driving behavior. Using this model, we explore the effects of system phase transitions and different path choices on system congestion. The description of the proposed model will first be elaborated in Section 2. We will then present the results in Section 3, and close the paper with conclusion and some discussions on future work in Section 4

\section{DESCRIPTION OF THE MODEL}

The developed two-layer CA approach is described in this section. Suppose we have a two-dimensional $L \times L$ square lattice with periodic boundary. Each cell is labelled by two coordinates, $x$ and $y$, which represent its positions. In this model, each cell (at each layer) can only be occupied by one car. The two-layer concept can be perceived as combining two BML models. As mentioned, the BML model can only assume the vehicle moving to the right and in a downward direction. In this two-layer approach, each layer is bi-directional, as illustrated in Figure 1. In this setting, we refer to the layers as the positive and negative layers. In the positive layer, the vehicle can move down or move to the right; in the negative layer, the vehicle can move up or move to the left. To distinguish between these two layers in the model, we use a layer indicator $a$, where $a=-1$ for the negative layer and $a=1$ for the positive layer.

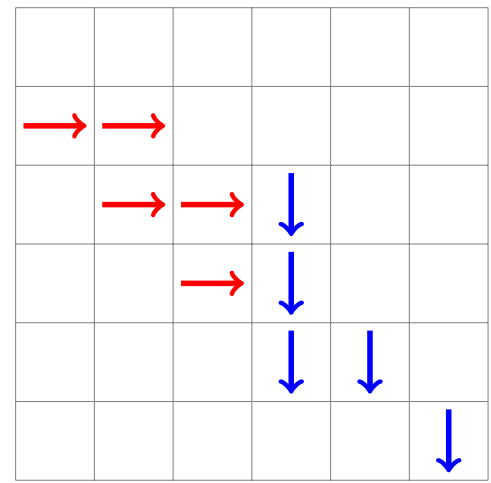

(a) Positive layer

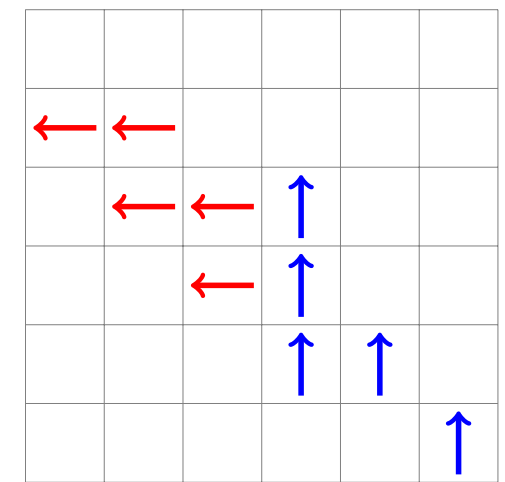

(b) Negative layer.

Figure 1. The positive and negative layers in the two-layer network model, where each layer is bi-directional.

To run the model, we first need to specify the size of the square lattice $L$ and the vehicle density $\rho$. We then randomly generate $N$ vehicles and their corresponding origin-destination pairs in the network, where $N=2 \rho L^{2}$, where the factor 2 is required to represent the two layers. The vehicle can only move to its neighboring grid, where the definition of the neighbor is based on the Von Neumann model, which was also used by Kennedy and Mendes (2006). The coordinate positions in $x$ (lateral) and $y$ (longitudinal) directions are defined for each vehicle. The position of the $i$-th vehicle at time $t$ is therefore denoted as $\left(x_{i}^{t}, y_{i}^{t}, a\right)$, where the layer is also specified by $a$. To mimic the real situation of speed limit, we set that the vehicle velocity is always less than the maximum velocity, $v \leq v_{\max }$. Another operating rule is that the vehicle can not move to a cell when it is occupied (within the same layer). The vehicles will then travel to the next position $\left(x_{i}^{t+1}, y_{i}^{t+1}, a\right)$ by following these operating rules at time $t+1$. Vehicles in the system will continue to travel following to the moving strategies, which will be described next, until they reach their destinations. The physical characteristics of the system are then assessed based on the average speed of the system, which will be further elaborated in Section 3. The moving strategies for the network model will be described next, followed by the descriptions of the quantities of interest as model outputs.

\subsection{Moving Strategy}

In this model, vehicles can only move from the current position to the neighboring position, the neighbor is defined as: $\left(x_{\text {neighbor }}, y_{\text {neighbor }}\right)=(x, y):\left|x-x_{\text {neighbor }}\right|+\left|y-y_{\text {neighbor }}\right| \leq r$. Here, $r$ is defined as the pitch distance measured between the midpoints of two adjacent cells. In other words, the vehicle can only move one cell at a time in the right or downward direction (when $a=1$ ) and left or upward direction (when $a=-1$ ). For 
illustration purpose, here we assume $v_{\max }=1$, i.e., there is no acceleration or deceleration as the vehicle can move, at most, one cell at each time step. First, let's define the origin positions of the vehicles as $\left\{x_{o_{i}}, y_{o_{i}}\right\}$, where $i \in(1,2, \ldots, N)$. Similarly, the positions of the destinations are denoted as $\left\{x_{d_{i}}, y_{d_{i}}\right\} \forall i$. At every time step, the intended movement is decided by comparing the current position to the destination:

$$
\left(\Delta x_{i}^{t}, \Delta y_{i}^{t}\right)=\left\{\begin{array}{l}
\Delta x_{i}^{t}=\operatorname{sign}\left(x_{d_{i}}-x_{i}\right) * \operatorname{sign}\left(\frac{2}{L}-\left|x_{d_{i}}-x_{i}\right|\right) \\
\Delta y_{i}^{t}=\operatorname{sign}\left(y_{d_{i}}-y_{i}\right) * \operatorname{sign}\left(\frac{2}{L}-\left|y_{d_{i}}-y_{i}\right|\right),
\end{array}\right.
$$

where $\operatorname{sign}(x)$ can have the value of $-1,0$, or 1 . In Equation 1, the first term signifies the direction under consideration, and the second term considers the influence of the periodic boundary. Since the algorithm only allows the vehicle to move one step (either lateral or longitudinal) at a time, we randomly select the direction of movement when both intended movement directions are non-zero. In other words, when $\Delta x_{i}^{t} \neq 0$ and $\Delta y_{i}^{t} \neq 0$ :

$$
\left(\widetilde{\Delta x}_{i}^{t}, \widetilde{\Delta y}_{i}^{t}\right)= \begin{cases}\left(\Delta x_{i}^{t}, 0\right) & \text { if } p \geq 0.5 \\ \left(0, \Delta y_{i}^{t}\right) & \text { otherwise }\end{cases}
$$

where $p$ is a random number between $[0,1]$, which is assumed to be uniformly distributed. As shown in Equation 2, an equal probability is assumed between the $x$ and $y$ directions. When one of the intended movement direction is zero, $\left(\widetilde{\Delta x}_{i}^{t}, \widetilde{\Delta}_{i}^{t}\right)=\left(\Delta x_{i}^{t}, \Delta y_{i}^{t}\right)$. With the moving direction is finalized, we can then determine the appropriate layer $\left(a_{i}^{t+1}\right)$. To complete the moving strategy, the cell occupancy needs to be determined, since each cell can only be occupied by at most one vehicle. The variable $\lambda_{i}^{t}$ is set to 0 if the site corresponding to the next intended movement is already occupied by another vehicle, and 1 otherwise:

$$
\lambda_{i}^{t}= \begin{cases}0 & \text { if }\left(x_{i}^{t}, y_{i}^{t}\right)+a_{i}^{t+1}=\left(x_{j}^{t}, y_{j}^{t}\right), \exists j \\ 1 & \text { otherwise }\end{cases}
$$

With the moving direction and $\lambda_{i}^{t}$ defined, we can then express the next location (at time $t+1$ ) for each vehicle as:

$$
\left(x_{i}^{t+1}, y_{i}^{t+1}\right)=\left(x_{i}^{t}, y_{i}^{t}\right)+\lambda_{i}^{t}\left(\widetilde{\Delta x}_{i}^{t}, \widetilde{\Delta y}_{i}^{t}\right)
$$

To incorporate driver behavior, we introduce a flexibility index $f: 0 \leqslant f \leqslant 1$, which indicates the probability of a vehicle moving in a direction that is different from the that determined in Equation 2. When $f>0$ and $\lambda_{i}^{t}=0$, the driver can choose a different direction, hence a new $\left(\widetilde{\Delta x}_{i}^{t}, \widetilde{\Delta y}_{i}^{t}\right)$, with a probability $f$. The new direction is determined by evaluating the occupancy of other neighboring cells. Here, we assume that a "flexible driver" will tend to move to an empty spot, when the initial intended moving direction is blocked.

\subsection{Quantities of interest}

Due to the randomness involved in this algorithm, a certain number of simulations are required before it reaches a steady state. Let's suppose the simulation starts at $t=0$, the steady state solution is reached at time $t=T_{s}$. The maximum number of iterations is set to be $T_{e}$. Once finished, we can obtain the following output quantities, as a function of $L$ and $\rho$ : the average velocity $\bar{v}$, the total number of vehicles that reach their destinations, the average travel time, and the traffic flux. Flux, denoted as $\phi$, is an important variable that characterizes a traffic network. The flux is computed as $\phi=\rho * v$, and it represents the amount of traffic flow in the network. The system is defined as in a free flow state when flux increases as $\rho$ increases; otherwise, the system is in a congestion state.

\section{RESUlts AND Discussion}

We demonstrate the proposed method and discuss the results. To avoid bias, 100000 simulations are performed with random origin-destination pairs of the vehicles. The traffic simulation follows the operating rules and moving strategies as presented in Section 2. To see the effects of flexibility, four $f$ values are considered: $f=0,0.3,0.5,1$, and the results are compared. The first and the last cases refer to the cases without and with flexibility, respectively. 
Figure 2 illustrates the relation between $\rho$ and $\bar{v}$ for the different $f$ values. We can observe that as $\rho$ increases, $\bar{v}$ decreases, where the trend is smoother as the lattice size grows larger. The results also show that a phase transition exists for all cases. Here, a phase transition is defined as the change in the traffic network behavior, from the free-flow state to the congestion state. Visually, it is indicated by the sharp drop in $\bar{v}$, where the velocity becomes more sensitive with the increasing $\rho$. The density where the phase transition occurs is denoted as $\rho_{c}$. As the density increases past $\rho_{c}$, only moderate changes in $\bar{v}$ are observed. When a higher flexibility is assumed in the drivers' behavior, the phase transition is shifted to a higher $\rho_{c}$ value. This result suggests that as drivers tend to deviate from the shortest-path routes, the average speed is higher. In other words, they can reach the destination faster albeit the increase in the total travel distance. These observations are deemed consistent with the real traffic situation.
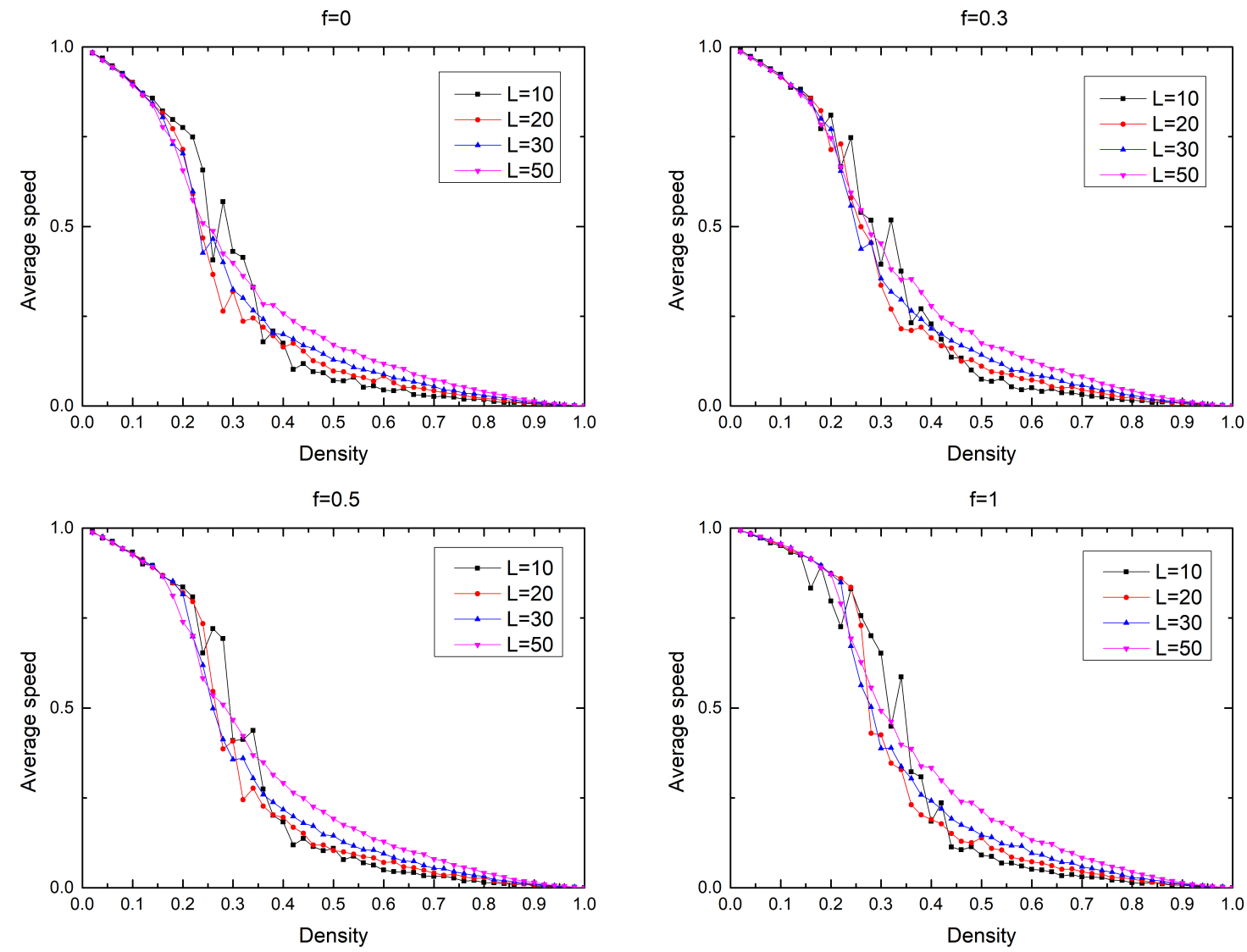

Figure 2. The simulation results of average speed as a function of density on square lattices with varying sizes.

The flux comparison is presented in Figure 3. As we can see here, a linear relationship between $\rho$ and $\phi$ is observed at the free-flow state. As density increases, flux also increases until it reaches a peak at $\rho \approx 0.2$, which corresponds to the phase-transition shown in Figure 2. Past $\rho_{c}$, the vehicle flux notably decreases despite the increase in density, which corresponds to the decreasing $\bar{v}$.

To validate the effectiveness of flexibility, we investigate the number of completed journeys and the total travel distance of the $N$ vehicles generated in the simulation. The number of completed journeys is obtained by keeping track of the vehicles that have reached their specified destinations. The travel distance for each vehicle is obtained by counting the total number of steps required to reach the destination from the origin point.

We can observe from Figure 4 that flexibility does not have any notable impacts on the traffic network when the traffic is very empty or very crowded. In between, however, both the total travel distance and the number of completed journeys increase notably as flexibility increases. Similar trends are observed in the two plots. 
B. Zhu and R. P. Liem, Routing Strategy Using Local Information ...

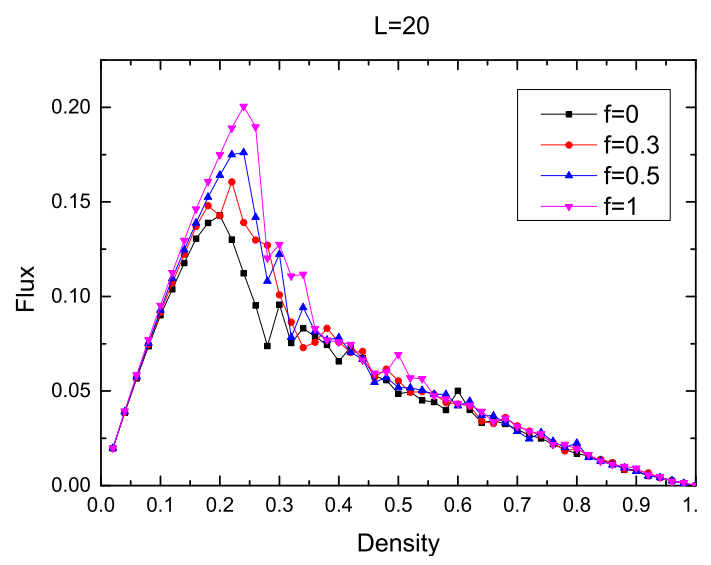

Figure 3. The simulation results of flux as a function of density on a square lattice with $L=20$, for varying flexibility values.

To explain this phenomenon, we first look at Figure 2. When $f=0, \bar{v}$ starts decreasing at around $\rho=0.15$, while the starting point of the phase transition when $f=1$ is shifted to $\rho=0.2$; these are consistent with the observation from Figure 4 . That is, when the road is almost empty or highly congested, the drivers' decisions will have insignificant impacts on the traffic condition. At other traffic densities, however, being more flexible to choose an empty road when a congestion occurs, instead of following the predefined shortest path, will result in a faster travel. This result is consistent with the real traffic situation.
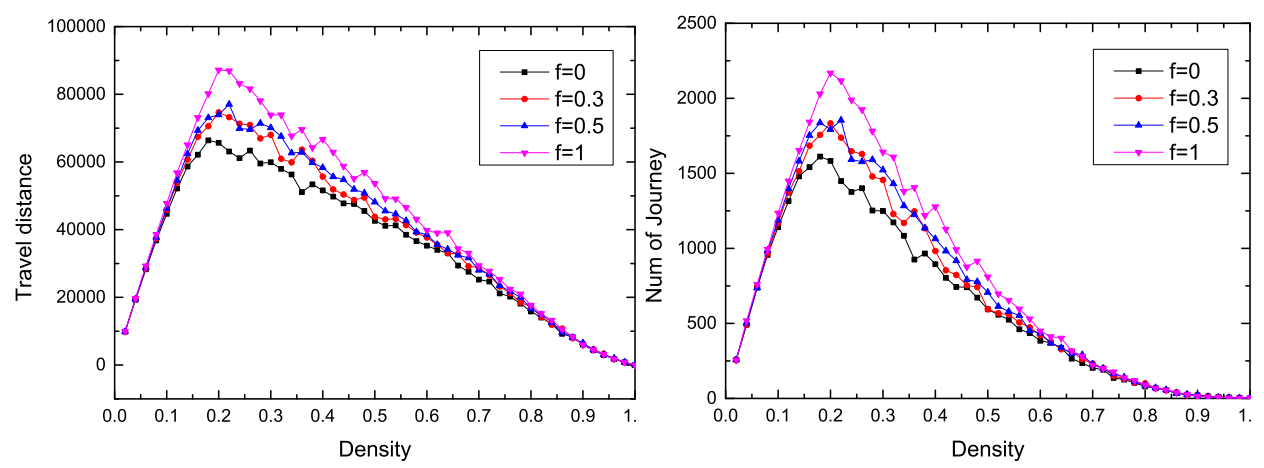

Figure 4. The simulation results of number of completed journeys and the total distance traveled as a function of density on square lattices with $L=50$.

\section{CONCLUSION AND FUTURE OUTLOOKS}

In this work, we developed a two-layer CA traffic model to study the characteristics of city traffic. This novel approach extended the capability of the commonly-used BML model from modeling two traffic directions to four, while providing the mechanism to avoid gridlock. With this improvement, the developed traffic models could better represent the actual car traffic situations, which enabled performing more realistic analyses on traffic networks. In addition, we also incorporated a flexibility index $(f \in[0,1])$ that reflected driving behavior into the model. This index denoted how likely would the drivers deviate from the shortest path when congestion occurred. When no flexibility was assumed $(f=0)$, drivers would always stick to the predefined shortest path regardless of the situation. When $f>0$, on the other hand, the driver might opt to take another path when the original path was blocked. Our results showed that introducing some degree of flexibility would shift the phase transition to a larger density value, thus delaying the onset of congestion. The phase transition occurred when the vehicle's average speed became very sensitive of the density, and dropped significantly as density increased. We also observed the benefits of being flexible within a certain range of densities, particularly in 
B. Zhu and R. P. Liem, Routing Strategy Using Local Information ...

terms of the total of completed journeys and the total distance traveled. In other words, when drivers were flexible, they could reach the destination faster albeit covering a longer traveling distance. This phenomenon, however, was not observed when the traffic was really empty or really crowded. Moving forward, we would model the traffic network with cross sections to better mimic the actual road system, which could be done by modifying the square lattice system. The same approach would also be employed to study how traffic disruptions and accidents affected the overall traffic flow. Furthermore, we would extend the model to assess the global routing strategy, instead of relying only on the local information in making the moving decision.

\section{ACKNOWLEDGEMENT}

This paper is supported by the Hong Kong University of Science and Technology (HKUST) Initiation Grant (Grant No. R9354). The authors would like to thank the generous supports.

\section{REFERENCES}

Angel, O., A. Holroyd, J. Martin, et al. (2005). The jammed phase of the biham-middleton-levine traffic model. Electronic Communications in Probability 10, 167-178.

Burstedde, C., K. Klauck, A. Schadschneider, and J. Zittartz (2001). Simulation of pedestrian dynamics using a two-dimensional cellular automaton. Physica A: Statistical Mechanics and its Applications 295(3-4), 507-525.

Chen, S., W. Huang, C. Cattani, and G. Altieri (2012). Traffic dynamics on complex networks: a survey. Mathematical Problems in Engineering 2012.

Chopard, B. (2012). Cellular automata modeling of physical systems. Springer.

Davis, L. (2004). Multilane simulations of traffic phases. Physical Review E 69(1), 016108.

Golledge, R. G. (1995). Path selection and route preference in human navigation: A progress report. In International Conference on Spatial Information Theory, pp. 207-222. Springer.

Kennedy, J. and R. Mendes (2006). Neighborhood topologies in fully informed and best-of-neighborhood particle swarms. IEEE Transactions on Systems, Man, and Cybernetics, Part C (Applications and Reviews) 36(4), 515-519.

Li, X.-G., B. Jia, Z.-Y. Gao, and R. Jiang (2006). A realistic two-lane cellular automata traffic model considering aggressive lane-changing behavior of fast vehicle. Physica A: Statistical Mechanics and its Applications 367, 479-486.

Maerivoet, S. and B. De Moor (2005). Cellular automata models of road traffic. Physics reports 419(1), 1-64.

Nagatani, T. (1993). Effect of traffic accident on jamming transition in traffic-flow model. Journal of Physics A: Mathematical and General 26(19), L1015.

Oliveira, E. M. R. and A. Carneiro (2014). Routine-based network deployment. In 2014 IEEE Conference on Computer Communications Workshops (INFOCOM WKSHPS), pp. 183-184. IEEE.

Tang, T., H. Huang, S. Wong, and R. Jiang (2007). Lane changing analysis for two-lane traffic flow. Acta Mechanica Sinica 23(1), 49-54.

Tang, T.-Q., X.-F. Luo, J. Zhang, and L. Chen (2018). Modeling electric bicycles lane-changing and retrograde behaviors. Physica A: Statistical Mechanics and its Applications 490, 1377-1386.

Wagner, P., K. Nagel, and D. E. Wolf (1997). Realistic multi-lane traffic rules for cellular automata. Physica A: Statistical Mechanics and its Applications 234(3-4), 687-698.

Weifeng, F., Y. Lizhong, and F. Weicheng (2003). Simulation of bi-direction pedestrian movement using a cellular automata model. Physica A: Statistical Mechanics and its Applications 321(3-4), 633-640.

Yeung, C. H. and D. Saad (2012). Competition for shortest paths on sparse graphs. Physical review letters 108(20), 208701.

Zamith, M., R. C. P. Leal-Toledo, E. Clua, E. M. Toledo, and G. V. de Magalhães (2015). A new stochastic cellular automata model for traffic flow simulation with drivers behavior prediction. Journal of computational science 9, 51-56.

Zheng, Z. (2014). Recent developments and research needs in modeling lane changing. Transportation research part B: methodological 60, 16-32. 